\title{
An internal splash: Levitation of falling spheres in stratified fluids
}

\author{
Nicole Abaid and David Adalsteinsson \\ Department of Mathematics, University of North Carolina, Chapel Hill, North Carolina 27599
}

\author{
Akua Agyapong \\ Hampton University, Hampton, Virginia 23668 \\ Richard M. McLaughlin a) \\ Department of Mathematics, University of North Carolina, Chapel Hill, North Carolina 27599
}

(Received 22 April 2003; accepted 26 January 2004; published online 5 April 2004)

\begin{abstract}
We experimentally explore the motion of falling spheres in strongly stratified fluids in which the fluid transitions from low density at the top to high density at the bottom and document an internal splash in which the falling sphere may reverse its direction of motion (from falling, to rising, to falling again) as it penetrates a region of strong density transition. We present measurements of the sphere's velocity and exhibit nonmonotonic sphere velocity profiles connecting the maximum and minimum terminal velocities, matching earlier measurements [J. Fluid Mech. 381, 175 (1999)], but further exhibit the new levitation phenomenon. We give a physical explanation of this motion which necessarily couples the sphere motion with the stratified fluid, and vice versa, and supplement this with a simplified, reduced mathematical model involving a nonlinear system of ordinary differential equations which captures the nonmonotonic transition and agrees with the measured velocity profiles at all depths except those in the vicinity of the sharp transition for which the model deviates from the measured speeds. We repeat the experiments adjusting the distance between the camera and falling sphere thereby reducing the optical blur associated with the change in optical refractive index associated with the strong density transition. By directly measuring the residual optical distortion with a center plane, vertical ruler, we exhibit that the measured velocity profile within the transition layer is strongly sensitive to the details of the measured optical distortion, and show subsequent improved agreement between the measurement and the model. Through direct measurement of the nonlinear mapping between physical and imaged coordinates we document measured velocity error trends which may occur from inaccurately accounting for this optical distortion. We suggest strategies for correcting this localized measurement detail generally. (C) 2004 American Institute of Physics. [DOI: 10.1063/1.1687685]
\end{abstract}

\section{INTRODUCTION}

The interaction between a solid body and a surrounding fluid is a common and important occurrence familiar at some level to everyone. From early days, we are confounded with the problem of understanding the drag a moving object experiences, and complexities associated with the drag crisis, and ensuing practical and useful ramifications, such as dimpled golf balls, improved aerodynamics, curve balls, just to mention a few important real world applications. ${ }^{1,2,3}$ The nature of this interaction dictates the modes of propulsion which organisms of different sizes use to move themselves around, and it has even been suggested that the shape evolution of various organisms has been strongly influenced by the particular range of the nonlinear and nonmonotonic drag law a particular species samples.

One natural goal is to deduce, either empirically, or in special cases ${ }^{3,4}$ from first principles, the drag law giving the functional relation between the speed of the body and associated drag the body experiences. Elementary dimensional

\footnotetext{
a) Author to whom correspondence should be addressed. Electronic mail: rmm@amath.unc.edu
}

scaling arguments show us quickly that at low speeds, we have Stoke's flow, in which the drag is linearly proportional to the speed, whereas at high speeds, inertial effects dominate and the drag is quadratically proportional to speed, ${ }^{3}$ and in between involves complex phenomena associated with turbulent boundary layers exhibiting remarkable nonmonotonic dependence of the drag law upon the speed. Nonetheless, having such a law represents a tremendous simplification; rather than having to characterize all the degrees of freedom associated with the surrounding fluid, the effect of all these variables (which may embody complex turbulent motions, etc.), is lumped into a single drag law.

Here, we focus upon a fluid system in which the fluid variables themselves become active degrees of freedom, namely, a strongly stratified fluid. Stratified fluids are those in which the background, equilibrium density field varies with height. Such fluids are ubiquitous in nature. Examples include our atmosphere over large vertical scales, in atmospheric boundary layers, and in the thermoclines of lakes and oceans. Much work has focused upon many fundamental, sociologically, and environmentally relevant problems. ${ }^{5-11}$ Here we will examine a seemingly simpler problem of predicting the motion of a sphere, falling under the influence of 
gravity through a stably (salt) stratified transition layer. Relatively few studies of such problems exist in the literature. Larsen explore the motion of a displaced, but neutrally buoyant bead in the presence of a linearly stratified medium and examined correlations between the bead motion and the generated internal wave field. ${ }^{12}$ The work of Srdic-Mitrovic, Mohamed, and Fernando explored the motion of dense settling particles through density transition layers, and identified a boundary layer shedding phenomenon associated with the slowing down of the particles below the terminal velocities of the lower layer, and additionally documents that the generated internal wave field plays a secondary role in the bead dynamics. ${ }^{13}$ Torres, Hanazaki, Ochoa, Castillo, and Van Woert numerically explored the flow induced by the vertical motion of a sphere in a linearly stratified medium, and focussed upon the properties of the collapse of the standing vortex at moderate Reynolds numbers. ${ }^{14}$

Our experimental results document the possibility of the coupling between the fluid and sphere to be sufficiently strong in some regimes to result in transient bead levitation. We will see that the presence of stratification endows fluid parcels with a potential energy which necessarily must be incorporated as a degree of freedom to correctly predict the sphere's motion through a strong density transition. We exhibit that the sphere's velocity undergoes a nonmonotonic connection between the higher terminal velocities at high altitudes and the slower terminal speeds at lower altitudes (on account of the smaller density difference between the descending sphere and background density field at lower altitudes). While similar nonmonotonic velocity profiles for spherical particles settling in stratified environments have been previously observed, ${ }^{13}$ surprisingly, we show here that the sphere may in fact "splash" off an internal layer of strong density transition, and reverse its direction of motion, hovering for some time in the vicinity of the transition before ultimately returning to its decent. In other words, the depth of the sphere itself is a nonmonotonic function of time. This is especially surprising in that the splash is occurring in the vicinity of a continuously stratified density profile, in the complete absence of surface tension. We argue that the entrained fluid viscously coupled to the sphere may develop its own negative potential energy (buoyancy reversal), and we present a nonlinear dynamical system modeling this viscous coupling to an extra degree of freedom which successfully captures phenomena associated with the sphere's motion. We show that such phenomena is all about turbulent mixing and entrainment, and offers a setup towards improved understanding of environmental mixing.

The paper is organized as follows: In Sec. II, we present the experimental setup and measurement procedures. We document the density profiles studied, and present terminal velocity measurements for the array of spheres falling in constant density fluids. In Sec. III, we present measurements for the velocity profiles of the falling spheres in stratified fluids, and evidence for the physical mechanisms responsible for the interesting motion. We additionally explain an optical distortion arising from the high contrast change in refractive index across the layer, and show how to correct for it. In Sec. IV, we present and discuss a reduced, nonlinear dynamical

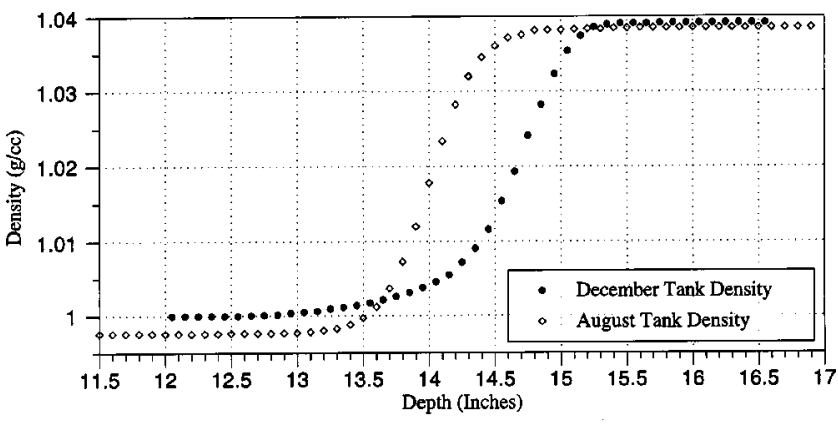

FIG. 1. Measured density profiles vs depth, using $\rho=0.997+0.00065 S$ $+0.000322(25-T) \mathrm{g} / \mathrm{cm}^{3}$ for salinity, $S$ in ppt, and temperature, $T$, in ${ }^{\circ} \mathrm{C}$.

system to model the sphere trajectories based upon a hydrodynamic coupling through the viscous drag law between the sphere body, and the surrounding stratified fluid. We exhibit that with a single adjustable parameter, the entrained fluid mass, this model fits the flight paths well for the entire array of beads studied.

\section{EXPERIMENTAL SETUP}

The experimental facility is a glass tank of dimension, ( $L=25$ in., $H=24.5$ in., $W=12$ in.). The tank is first halffilled with a well mixed, salt water solution, of density $\rho$ $=1.0385 \mathrm{~g} \mathrm{~cm}^{3}$, and then the tank is slowly filled with fresh water. Measurements are reported for different studies, the first performed in December 2002, and the second in August 2003. The ensuing density profile is measured before and after the experiment is performed by means of an Orion conductivity probe, and yields a strong density transition layer between depths of 14.25 in. and 15.25 in. for the December data, and between depths 13.5 in. and 14.5 in. for the August experiment, as shown in Fig. 1, with depth measured from top to bottom, connecting the top fresh water layer to the bottom dense brine layer. We note our density fit in the caption which was obtained using a digital scale accurate to 0.01 $\mathrm{g}$, and $2000 \mathrm{ml}$ flask accurate to $0.5 \mathrm{ml}$. The probe was subsequently calibrated with respect to this fit, and used to measure salinity and temperature directly by dialing a calibrated Velmex slider slowly through the tank. Measurements were taken on $0.1 \mathrm{in}$. increments, starting at a depth of 11.5 in. and continuing uniformly through a depth of $17 \mathrm{in.} \mathrm{The}$ data are shown in Fig. 1. An array of spherical glass beads of mass $=0.06-0.07 \mathrm{~g}$, and approximate radius $=0.25 \mathrm{~cm}$ having precise density varying between $1.04 \mathrm{~g} / \mathrm{cm}^{3}$ (for precise mass, volume, density values, see Table I in Sec. IV below) and $1.1 \mathrm{~g} / \mathrm{cm}^{3}$ in increments of $0.005 \mathrm{~g} / \mathrm{cm}^{3}$ accurate to $0.0005 \mathrm{~g} / \mathrm{cm}^{3}$ are released from the top of the tank and the motion is recorded by means of a Photron FastCam PCI digital video camera using frame rate $60 \mathrm{fps}$ except in two cases in which we employ $30 \mathrm{fps}$. The profiles are slightly different for the two studies. In December, the fresh top layer is colder than utilized in the August tank, and the top layer density profile is slightly higher (top: $0.999 \mathrm{~g} / \mathrm{cm}^{3}$ for December vs $0.997 \mathrm{~g} / \mathrm{cm}^{3}$ for August), while the bottom layer is slightly saltier in August (bottom: $1.039 \mathrm{~g} / \mathrm{cm}^{3}$ for December vs $1.0385 \mathrm{~g} / \mathrm{cm}^{3}$ for August). The camera is positioned dif- 
ferently for the two studies: For the December 2002 study, the camera is positioned a distance of $30.75 \mathrm{in}$. from the front of the tank, at a height of $10.75 \mathrm{in}$. from the ground to center of lens (aligned with tank rulers at a depth of $15 \mathrm{in}$.), and horizontally $5 \mathrm{in}$. from the left end of the tank. For the August 2003 study, the camera is moved closer and a wider angle was employed so as to reduce the optical distortion from change in refractive index in the fluid occurring across the density step. In this case, the camera is positioned 18.25 in. from the front of the tank. The vertical plane on which the beads are dropped is varied between the two studies. For the December study, the beads are all released into the tank 9 in. from the left end of the tank, and precisely halfway between the front and backwalls of the tank. The beads are clearly sufficiently far from the walls to not experience any wall effects. In the August study, the beads are released 3 in. from the front wall so as to reduce the optical distortion. In the December study, the experiment is illuminated by a Sylvania Sun Gun II movie light positioned 14 in. from the front wall, at a height of $29.5 \mathrm{in}$., and horizontally $13 \mathrm{in}$. from the left end of the tank so as to capture a clear shadowgraph of the entrained fluid on the back wall of the tank. No additional light beyond ambient was used in the August study. Two methods of ruler alignment are employed as a means to extract the precise depth of the bead within the tank. Rulers are placed on the front wall and back wall of the tank to (through digital postprocessing) eliminate parallax distortion associated with the change in index of refraction, and to correct for the fixed position of the camera. The imaged falling body may be represented in pixel (image) coordinates. We employ two different techniques to define a mapping between the pixel coordinates, and the true depth coordinates. In the December study, this mapping is defined as follows: Measure the pixel coordinate over a range of depths on the front and back rulers, average this result, and perform a piecewise linear fit to this mapping connecting the two linear fits within the transition layer. The output of this procedure compares well to direct measurements taken with a third ruler inserted into the tank along the vertical midplane for depths outside of the transition layer, however, within the transition layer, the mapping deteriorates. An improved technique is employed for the August study. In that case, a carefully leveled third ruler is aligned with the front and back rulers and filmed on the 3 in. vertical plane on which the spheres are released. The ruler is carefully removed, and then the experiment is run. Digital images are then measured using this third ruler, which defines a nonlinear mapping from the pixel coordinates into the physical coordinates. For each image, we subtract the background, and find a suitable isocontour of the sphere in the resulting array. The sphere's center of mass is computed in pixel coordinates as a function of time, and mapped into the physical coordinates with one of the two previously outlined methods. The velocity is computed through time differentiating the result. The video is analyzed using the Macintosh software package, DataTank developed by one of the authors, Adalsteinsson.

On account of different bead densities, the Reynold's number (nondimensional number comparing inertia with viscous forces) and the Froude number (comparing inertia and

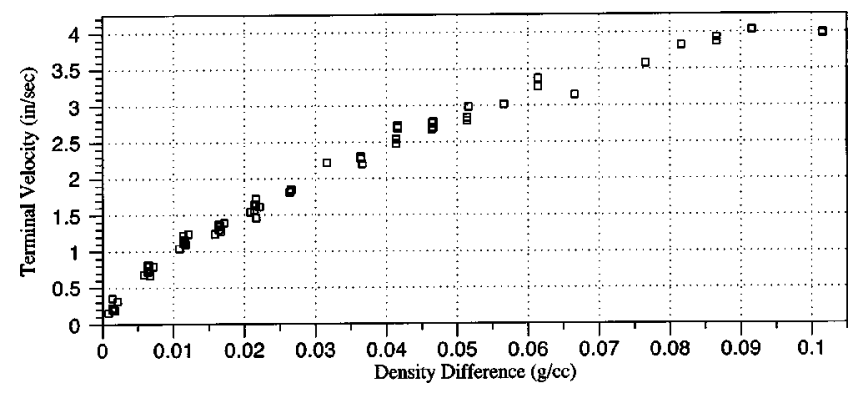

FIG. 2. Measured terminal velocity vs density difference between constant density tanks, and relative bead density.

buoyancy) vary in our experiments. For the characteristic scale based upon the bead diameter, $D$, and observed terminal velocities, $V$, the Reynolds number, $V D / \nu$ ranges from 20 to 450 , with $\nu$ being the kinematic viscosity of water. The Reynolds number of the stratified tank will be outside of these bound associated with the strong internal splash phenomena occurring there (and somewhat difficult to determine without utilizing a microscale). In our experiments, the Froude number, $\mathrm{Fr}=(U / D) \sqrt{L \rho / g \Delta \rho}$ ranges over the interval $(5,20)$ for characteristic velocities, $U$, taken as the terminal velocity of the top layer, $\rho$ is the density of the bottom layer, and $\Delta \rho$ is the change in tank density over distance, $L$. We note that on account of the lower terminal velocity of the bottom layer, a different range of Froude numbers may be defined by these lower velocities. It was through adjusting these lower terminal velocities that we discovered the transient levitation of these falling spheres.

To deduce the relevant drag law in constant density environments, the beads were first dropped into fresh water tanks, and their terminal velocities were directly measured using the DataTank program, and the results are presented in Fig. 2, showing terminal velocity as a function of density difference between the bead and background water. We varied the salinity of the constant density tank to enlarge the data set. The beads are not perfectly spherical, and consequently, we present this figure to document that the subsequent phenomena we present is not associated with a strong nonmonotonic drag law as occurring, say with phenomena associated with a drag crisis. The terminal velocities show a good functional fit with respect to the density difference

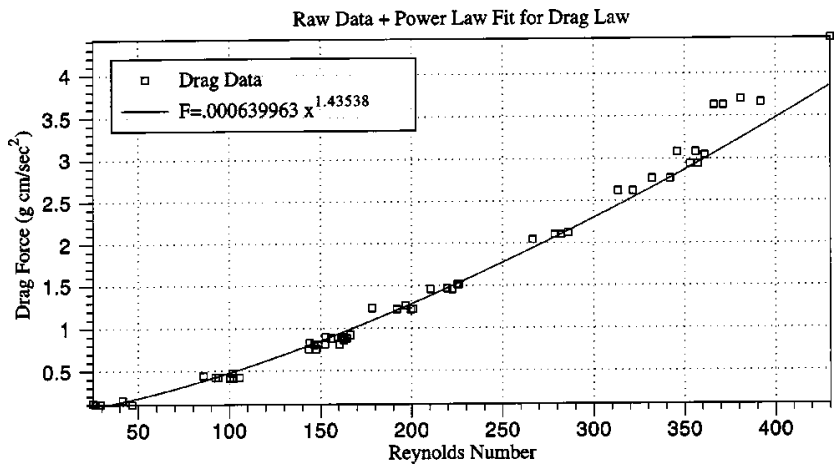

FIG. 3. Drag force $\left(\mathrm{g} \mathrm{cm} / \mathrm{s}^{2}\right)$ obtained from reduced gravitational force (see text), vs Reynolds number. 

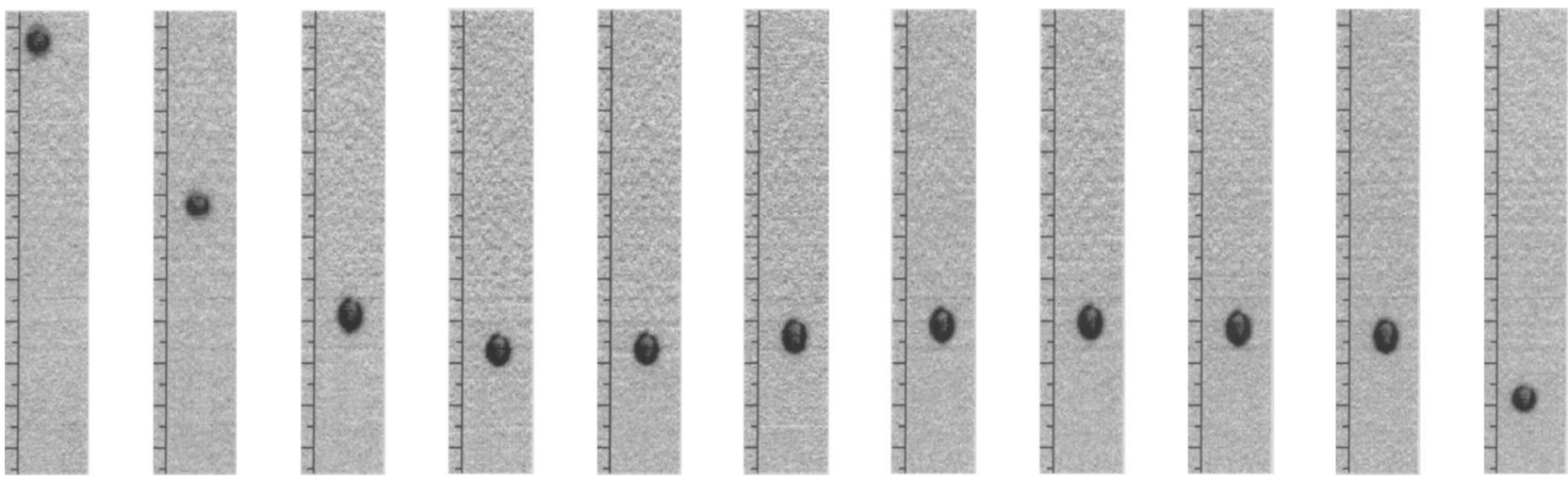

FIG. 4. Digital snapshots of bead position at different times, the leftmost image at $t=0.8 \mathrm{~s}$, each image stepping in increments of $0.5 \mathrm{~s}$, except the final image which jumps to final snapshot of the experiment, taken at $t=35 \mathrm{~s}$.

(showing good similarity between the different spheres), however, since each bead has different mass, and different volume, we use these data to obtain a power law fit for the drag force as a function of the Reynolds number. The drag force is measured through the gravitational force, $m g \rho^{\prime} / \rho_{b}$, where $\rho_{b}$ denotes the bead density, and $\rho^{\prime}$ is the difference between the bead density and water density. The data and subsequent fit are shown Fig. 3.

\section{TRAJECTORY MEASUREMENTS IN A STRATIFIED FLUID}

We return now to the case involving the stratified fluid with background density profile documented in Fig. 1, and explore the motion of an array of different specific gravity glass spheres falling through this medium. In this section we make five observations. First, we experimentally document the transient levitation event in which the sphere motion switches from falling to rising, carefully documenting the phenomena to be nonoptical. Second, we experimentally document the key physical mechanism responsible for these phenomena which is the hydrodynamic coupling between the sphere body and ambient, stratified fluid. Third, we document a long lived attached boundary layer of upper layer fluid which produces an extraordinarily long transient time scale. Fourth, we present flight measurements for the array of different density spheres and document regimes in which this hydrodynamic coupling is most pronounced. Last, we explain the optical distortion occurring with the transition layer, and employ a method to correct the measurements.

In Fig. 4 we present snapshots of the tank focusing upon the bead as it penetrates the density transition layer. With the exception of the last image, each snapshot is taken at equal time increments, $\Delta t=0.5 \mathrm{~s}$, commencing at time, $t=0.8 \mathrm{~s}$; the last image is taken at $t=35 \mathrm{~s}$, to exhibit the ultimate, but remarkably slow descent of the sphere. Clearly, this sequence documents the reversal of motion of the sphere, as it falls into and through the layer, then changes direction, and commences to rise, before ultimately falling through to the bottom of the tank. We will return shortly to discussing the remarkably long transient and incredibly slow descent in the bottom layer, as compared with the expected terminal velocity of the bottom layer. The ruler depicted in the figure has smallest tickmarks of roughly 0.18 in., though should be taken as only a rough gauge of length, as no optical filtering is applied in this particular sequence. It is worth noting the strong optical distortion observable as the bead penetrates through the transition layer.

To document that this levitation phenomenon is not associated with the mild optical distortion observable in Fig. 4, we have repeated this same experiment, moving the descending sphere closer to the front wall (but still outside of the wall boundary layer) in the first case, and closer to the back wall in the second case). In Fig. 5, we present an analogous montage, only this time with the bead released 2 in. from the front wall, with displayed times shown on a half second interval, starting at $t=1 \mathrm{~s}$, except for the final two images, taken at $t=10 \mathrm{~s}$ and $t=15 \mathrm{~s}$, respectively. Observe, the optical distortion visible in Fig. 4 is essentially removed, whilst retaining a similar reversal of motion as the sphere exits the
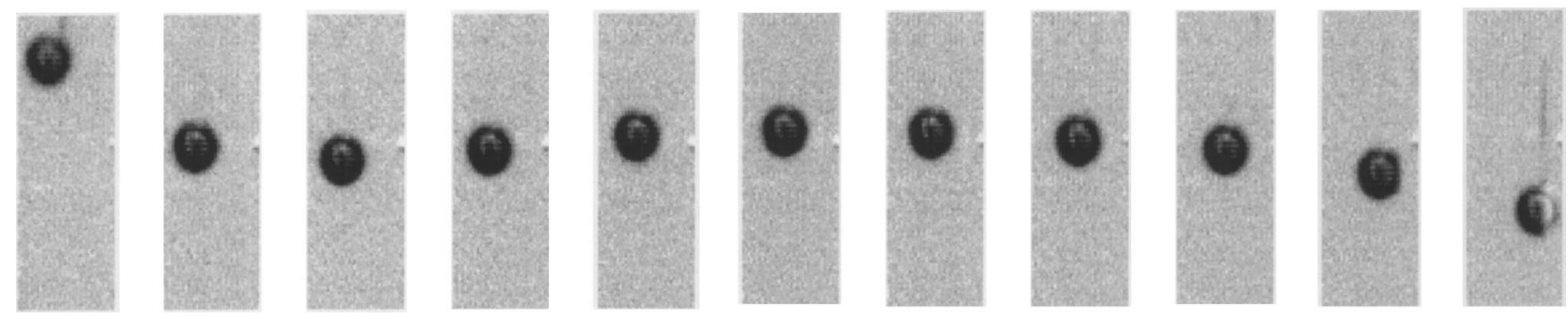

FIG. 5. Similar montage to Fig. 3, only bead released 2 in. from the front wall, with the leftmost image at $t=1 \mathrm{~s}$, each image stepping in increments of 0.5 $\mathrm{s}$, except the final two images at $t=10 \mathrm{~s}$ and $t=15 \mathrm{~s}$, respectively. 

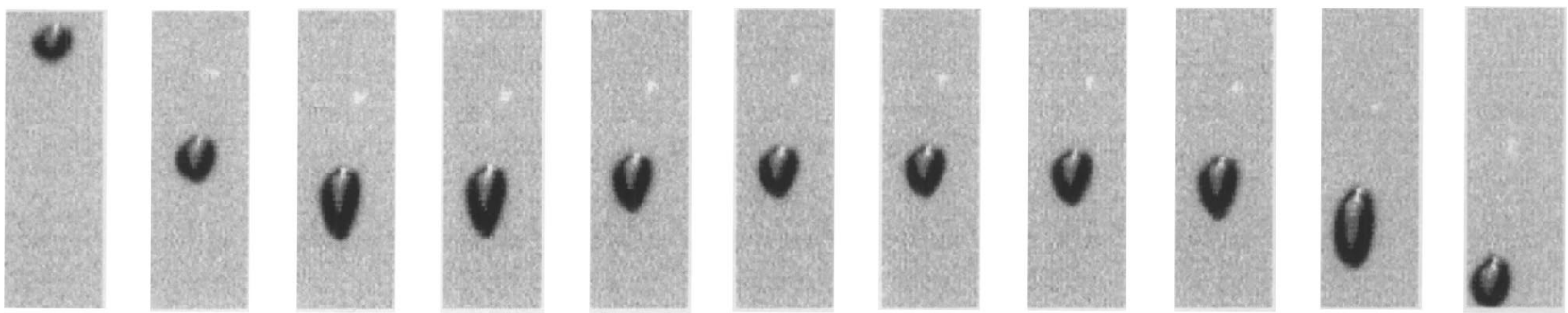

FIG. 6. Similar montage to Fig. 3, only bead released 2 in. from the back wall, with the leftmost image at $t=1 \mathrm{~s}$, each image stepping in increments of 0.5 $\mathrm{s}$, except the final two images at $t=10 \mathrm{~s}$, and $t=15 \mathrm{~s}$, respectively. Note the strong optical distortion.

transition layer. Shown in Fig. 6 is the same time sequence, only here the bead is released 2 in. from the back wall. We have kept the cropping area identical in this sequence, and have only moved the cropping region to track the bead path (of course, the distance to the camera is greater in this case by 8 in. as compared with the case presented in Fig. 5). Observe the dramatic optical distortion of the sphere as it moves through the transition region. Additionally, we note that the center of mass of the sphere moves in a pattern similar to the prior two cases, additionally documenting the reversal of motion as the sphere exits the transition region, albeit less satisfying than the case in Fig. 5 with no optical distortion. The optical distortion may be explained by geometric optics ray tracing, through a vertical index of refraction change in the stratified layer, along with the horizontal index of refraction change associated with the light ray passing from air to water (through the vertical glass wall). Consequently, the levitation phenomena is not attributed to optical distortion. For a digital online movie showing the moving sphere through this transition layer, please see Ref. 15. By moving the plane of motion closer to the front wall, complexities associated with the double index of refraction change may be substantially reduced, while retaining the identical reversal of motion. At the end of this section, in discussing the August 2003 data, we present an explanation for this optical distortion, and suggest a means for a correction.

It is worth remarking that an excellent study in a similar setup by Srdic-Mitrovic, Mohamed, and Fernando ${ }^{13}$ did not find the levitation phenomenon in which the bead motion switches from decent to ascent. The Reynolds numbers, and Froude numbers for that experiment, and our present experiment are quite comparable, and the possible explanations for the differences are threefold. First, the radii of the settling particles employed by Srdic-Mitrovic et al., were typically smaller than our beads by roughly one order of magnitude. The two different experiments have comparable length scales of background density transition, but in our experiment, the bead radius is comparable to the background density transition thickness. Second, the levitation phenomenon emerges as the buoyancy of the bead in the bottom layer is reduced; the Srdic-Mitrovic study focuses upon the Froude numbers set by terminal velocities of the top layers, and did not focus upon Froude numbers of the bottom layer, which is critical to adjust to observe the transient levitation. Last, the method of stratification in the two experiments is slightly different: In the Srdic-Mitrovic study, ethyl alcohol is added to the top layer to match optical indices of refraction, while in our study, our top layer was pure water. The diffusive properties of alcohol are different than that of salt, and consequently, the mixing properties of the entrained fluid are different than those of the present study.

Figure 7 shows the closeup shadowgraph snapshots of the bead as it enters and exits the transition layer. (This sequence is taken from a different trial than the previous case documented in Fig. 4, here exhibiting the bead arresting, but not rising.) These are taken at uniform times, on half second increments starting just before the entrained plume ascends
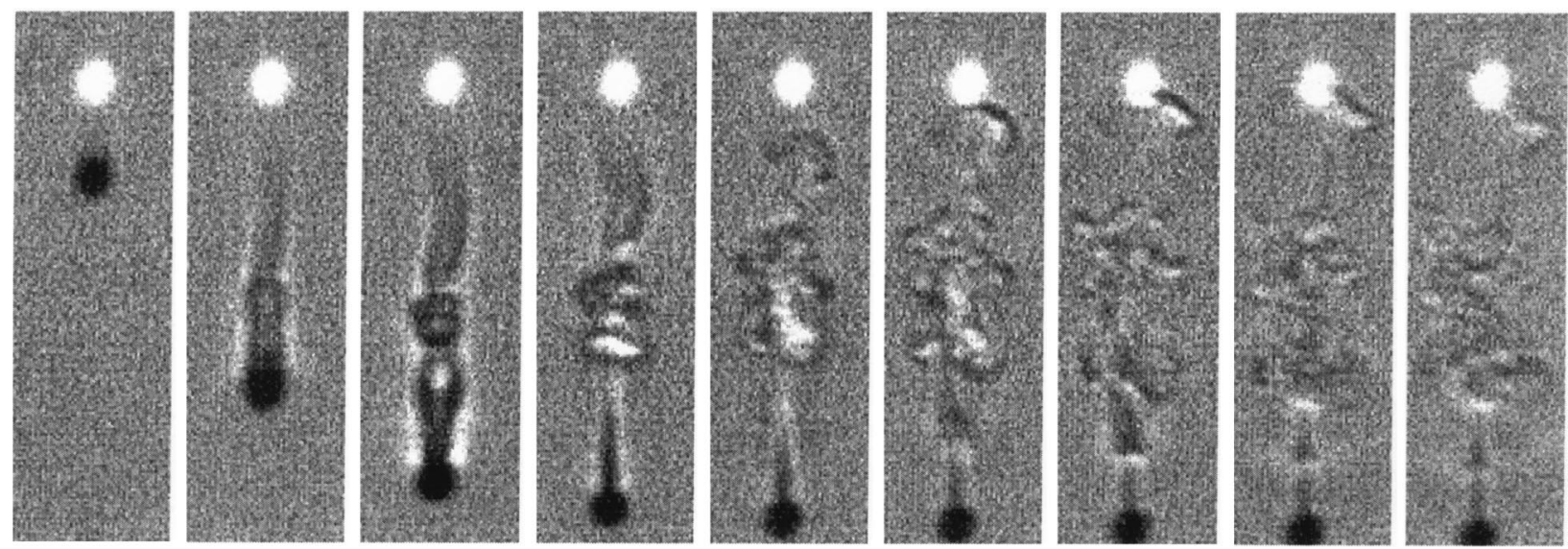

FIG. 7. Closeup snapshots of shadowgraph as the bead penetrates the transition layer at uniform hall second increments, depicting the entrained plume, and subsequent detachment. Observe the bead stops descending as the entrained fluid returns to the top layer. 

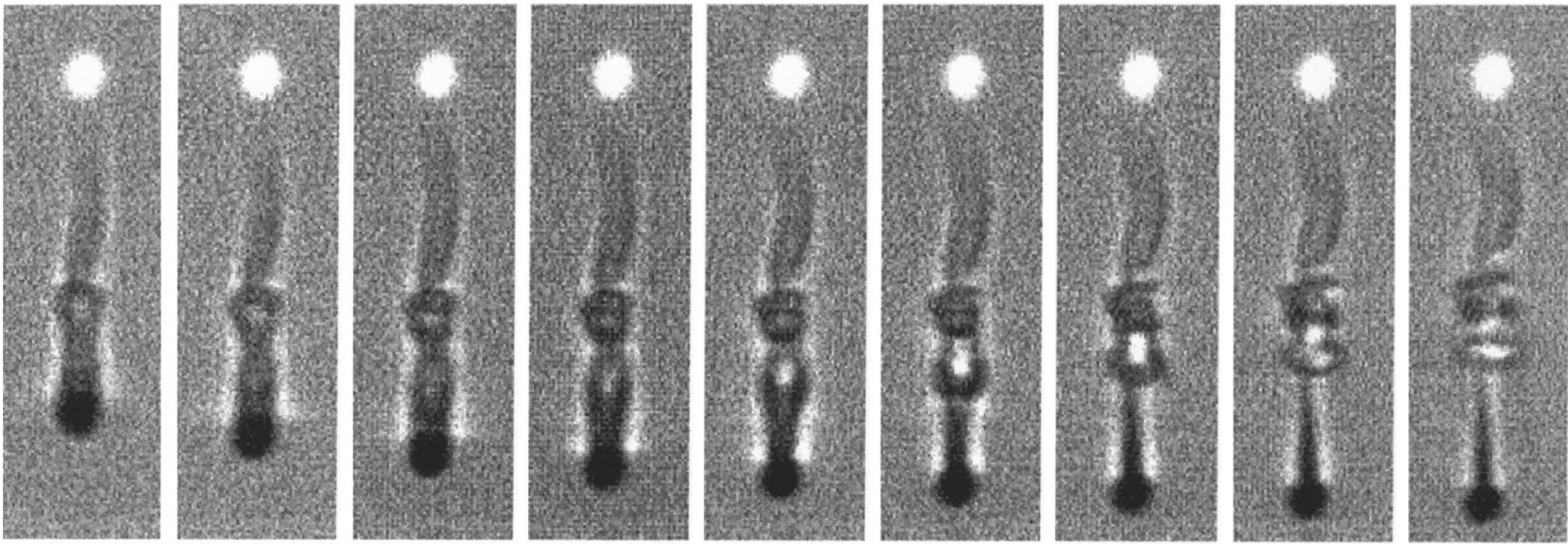

FIG. 8. Additional frames between frame 2 and frame 4 of Fig. 7 showing the dynamic reversal of motion of the entrained fluid.

back into the top layer. In Fig. 8, we show the temporal details of the plume evolution occurring between frame 3 and frame 4 of Fig. 7. For a digital movie showing the dynamic evolution of this plume, please see the EPAPS website. ${ }^{15}$ Observe, the entrained fluid is clearly visible in the shadowgraphs, and the time sequence documents the reverse motion of the entrained fluid relative to the bead as it falls through the transition layer. Inspection of the frame sequence shown in Fig. 8 shows that a mass of upper layer fluid is dragged some distance into the lower layer by the falling sphere, but this mass experiences a buoyancy reversal and clearly moves in a direction contrary to the falling object. This is an internal splash. This motion of the entrained fluid provides evidence for the key physical mechanism giving rise to the nonmonotonic (local minimum of the sphere's speed) connection between the different terminal velocities associated with the top and bottom layers. This plume additionally offers the natural interpretation of an internal "splash" phenomenon directly responsible for the beads subsequent (and temporary) change from descent to ascent. Observe, beyond this time, the bead motion switches from falling to actually rising. At longer times, the bead eventually returns to descending to the bottom of the tank.

\section{A. December 2002 measurements}

In Fig. 9, we present the digitally measured velocity profile vs depth for five different bead drops, curves from bottom to top ranging from $1.04 \mathrm{~g} / \mathrm{cm}^{3}$ to $1.06 \mathrm{~g} / \mathrm{cm}^{3}$ in increments of $0.005 \mathrm{~g} / \mathrm{cm}^{3}$ for the experiment performed in December 2002. These measurements are obtained using the package DataTank written by one of the authors, and all subsequent measurements are taken in a tank with one of the two density profiles shown above in Fig. 1. The fixed camera position and additional first order parallax distortions associated with the change in optical refractive index arising from the variable background density profile are removed by aligning and averaging the back and front rulers. The trend is clear: From the observed motion of the sphere, the velocity profile vs depth is a nonmonotonic connection between the terminal velocities of the upper and lower fluids. We emphasize that each of these beads have greater density than the fluid in the tank at all altitudes. Moreover, the smaller density beads experience a deeper minimum speed as compared with the terminal velocity of the bottom layer, and the 1.04 $\mathrm{g} / \mathrm{cm}^{3}$ bead even reverses its direction of motion and ascends for a transient time scale. We additionally emphasize another

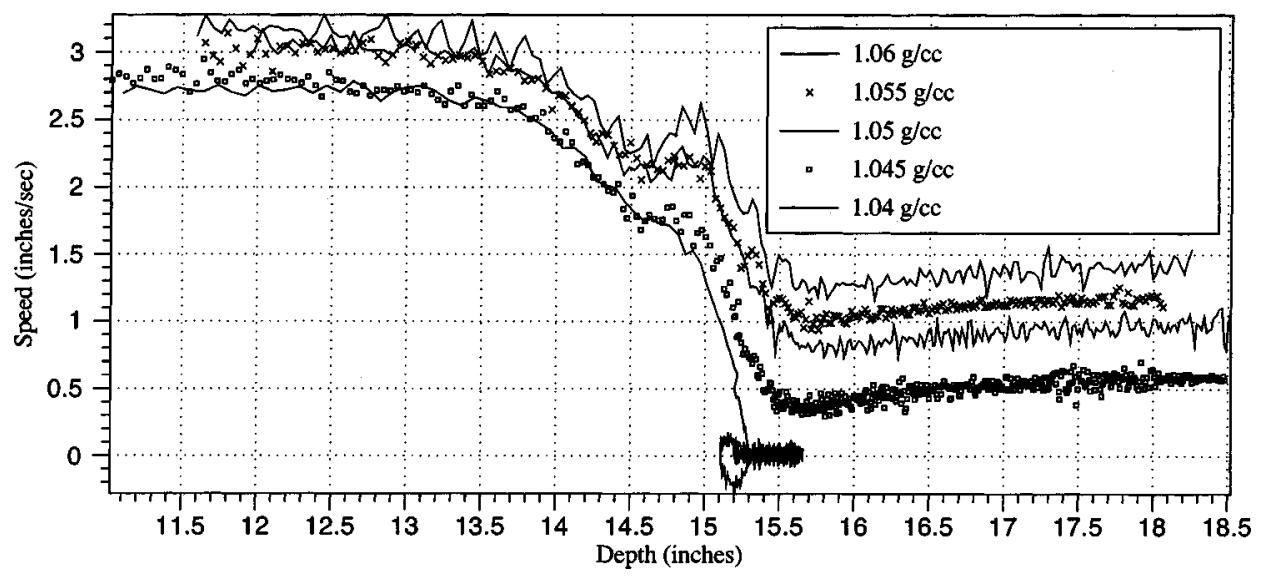

FIG. 9. Measured bead velocities in December 2002 stratified tank as a function of depth. 
remarkable phenomenon occurring in this experiment with the beads closer to neutral buoyancy in the bottom layer. This is an extraordinarily long transient time scale to reach the terminal velocity of the bottom layer for beads whose density is near the density of the bottom layer. The evidence for this behavior is the following: The tank was first filled to a depth of 14 in. with constant density salt water of density $1.039 \mathrm{~g} / \mathrm{cm}^{3}$. Before the fresh water was added to achieve the variable transition layer, we dropped each bead from rest to measure the terminal velocity exactly of the bottom layer for each bead in these tanks. Remarkably, the most neutrally buoyant beads, when dropped into the density stratified tanks, did not reach the terminal velocity of the bottom layer through the entirety of filmed motion through the tank. As an example, the terminal velocity of the $1.04 \mathrm{~g} / \mathrm{cm}^{3}$ bead in the $1.039 \mathrm{~g} / \mathrm{cm}^{3}$ constant density bottom layer in the December study was measured directly to be $0.17 \mathrm{in} . / \mathrm{s}$, however, after this bead passed through the transition layer in the stratified case, until the memory elapsed on the camera (at $30 \mathrm{fps}$, after $35 \mathrm{~s}$ ), the bead had progressed less than 1 in. beneath the transition layer in $30 \mathrm{~s}$. Contrast this with the analogous case in the initial constant density lower half tank, in which the same bead progressed 1 in. in $6 \mathrm{~s}$. The physics for this long transient is that once the plume of light fluid has shed, and the bead is moving at a very low speed, there still exists around the bead a small boundary layer of fresh water which diffuses exceptionally slowly on account of the very long diffusion of time of salt in water, and the absence of a strong turbulent diffusion in this slow flow state. We comment on this point further below in the modelling Sec. V, and should be contrasted with the findings of Srdic-Mitrovic et al. ${ }^{13}$ who report all caudal fluid to be disassociated from the falling sphere once the plume has detached from the solid body. We note that this transient time scale greatly shortens with increasing bead density on account of the greater mixing occurring with stronger bottom layer terminal velocities. We further note that this effect requires a very slow bottom layer terminal velocities. For example, during the August study, the bottom layer density is slightly higher than the December study (the $1.04 \mathrm{~g} / \mathrm{cm}^{3}$ sphere decended at $0.19 \mathrm{in} . / \mathrm{s}$ in a constant density tank with the same bottom layer density as the August stratified tank, faster than that in the December measurement), and after passing through the transition layer, the sphere did accelerate to its lower layer terminal velocity. This phenomenon is just one additionally complex mixing phenomenon associated with the motion of moving solid bodies through stratified fluids.

The conceptual explanation of the just documented phenomena is clear. The sphere descends through a constant density environment, and experiences a linear drag law producing a constant speed motion in the top layer. Of course, to account for this constant motion, the sphere necessarily drags, through viscous forces, a shell of entrained fluid. There is no potential energy cost associated with moving this entrained fluid in a constant density environment until the entrained fluid itself experiences a sudden change in its surroundings. As the bead passes through the density transition layer, the entrained (caudal) fluid suddenly becomes less dense than the surrounding fluid it has moved into. This sud- den change in background density endows the entrained fluid with a potential energy which is restored by the entrained fluid (or plume) moving back into the top layer. This sequence is well documented in Fig. 8. Of course, the upward motion of the entrained fluid necessarily, through viscous coupling, exerts an additional upward force upon the bead itself, yielding the strong reduction, and subsequent upward motion of the bead itself. It should be stressed that mixing, or lack of mixing, is key here as the entrained fluid is constantly mixing with its surroundings. It is necessary that this mixing be slow enough relative to the speed of motion so that a local buoyancy reversal may occur. If the mixing occurs instantaneously, the bead will experience no additional upward force beyond the standard Archimedian force balance. Without doubt, the magnitude of the bead's minimum speed gives an observable measuring the entrainment and mixing processes occurring within the entrained fluid parcel.

One additional remark regarding the parallax distortion which is evident in each curve between the depths of $14.5 \mathrm{in}$. and $15 \mathrm{in}$. This is the region of strongest density gradient, and the bead actually warps optically as it passes this layer (see, for example, Fig. 6). At the end of this section, we show the steps necessary to digitally correct for this optical blur. To reduce the actual blur, it is necessary to reduce the distance from the camera lens to the falling sphere. We next present data for the August 2003 measurements with this in mind.

\section{B. August 2003 measurements}

In Fig. 10, we show the analogous flight trajectory for the August 2003 experiments as those presented in Fig. 9 for the December study. We have employed two experimental differences and two data acquisition and analysis differences in collecting this data. First, as noted in Sec. II, the distance between the camera and falling body is reduced to minimize optical distortions. Second, also noted in Sec. II, we have intentionally decreased the density of the bottom layer by approximately $0.0005 \mathrm{~g} / \mathrm{cm}^{3}$. Third, in determining the true sphere depth from observed (pixel) bead depth, a third ruler was inserted into the tank before the experiment along the vertical plane on which the beads are dropped. This ruler is leveled, and aligned with respect to the front and back wall rulers at the camera lens height. The pixel (digital image) coordinates are then subsequently mapped to physical coordinates by digitally observing for a given image depth (pixel coordinate) the associated true depth as observed on the third ruler. Last, for the August experiments, the computation (within the DataTank program) of the sphere velocity is computed used a seven point least square interpolation to reduce noise (in the December study, the velocities were obtained with a central difference scheme, and no smoothing was applied). In general, the August data were less noisy than the December data primarily on account of the reduced distance to the objective.

Observe, the primary features of the August velocity profiles compare well with those from the December data, with one noteable difference: The behavior of the measured velocity profile within the transition layer is considerably 


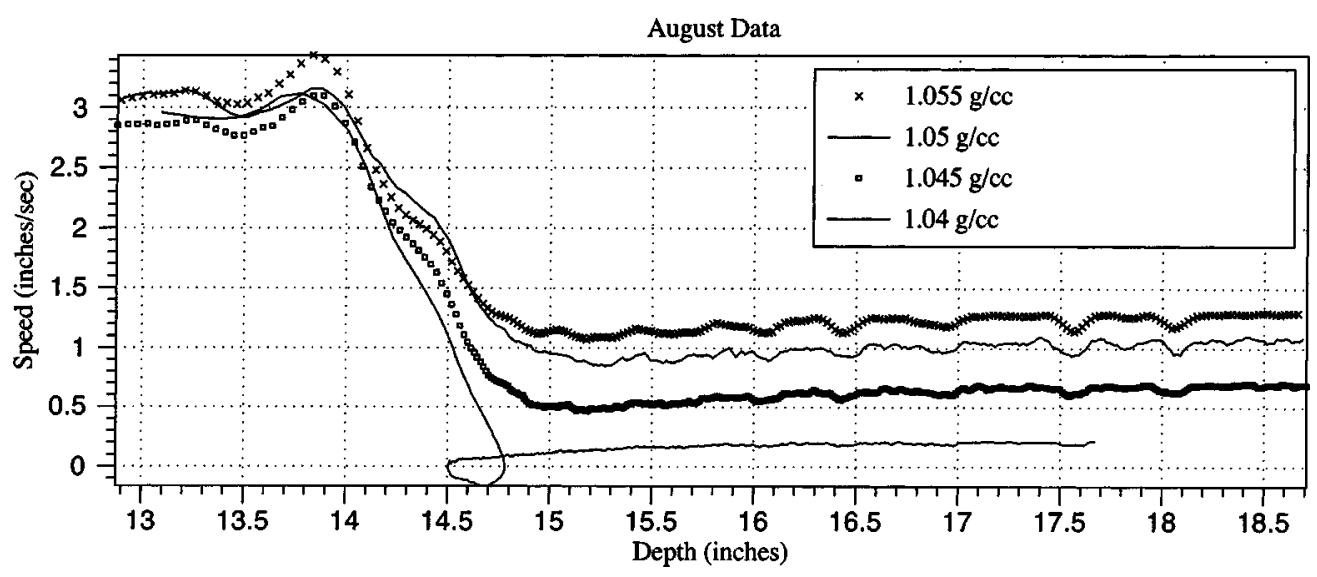

FIG. 10. Measured bead velocities in August 2003 stratified tank as a function of depth.

different. The general trend in the August data is an increase in the measured velocities within the transition layer as compared to the December data. The difference is optical, and is in how the data are collected. In the December data, the bead depth is determined through a piecewise linear averaging of the front and back wall rulers, whereas in the August data, the depth is deduced from the explicit (nonlinear) mapping of the pixel coordinates to real physical coordinates through the insertion of a third ruler. In Fig. 11, we compare the August $1.04 \mathrm{~g} / \mathrm{cm}^{3}$ measured velocity profile shown in Fig. 10 with a linear least squares fit between the true depth ruler and pixel coordinates (akin to that used in the December study). (The solid curve in the figure is the model output to be discussed later.) The triangles denote the data shown previously in Fig. 10, whereas the squares give the velocity profile measured using a linear fit to the mapping between physical and pixel coordinates, which is essentially the procedure used for the December measurements. Clearly, within the transition layer there is difference in the measurement: the linear fit for the pixel to physical space mapping yields a reduced velocity (squares) within the transition layer as compared with the nonlinear mapping (triangles).

To give a better visualization for this mapping, consider the following cartoon designed under the assumption of a sphere falling with a constant velocity in a variable density tank (which could be obtained through towing as opposed to free fall). To correct for the optical distortion, without perturbing the stratified layer too much, the following minimally invasive procedure is suggested to generally improve the measurements (which we employed during the August study). At the location where the bead will be dropped, we place a ruler (middle ruler) and align it with the front ruler. With the camera in the same location as will be used to film the beads dropping, we film the rulers. This third ruler is then calibrated by recording where the ruler marks on this temporary ruler are in terms of fixed pixels in the image. The top of Fig. 12 shows a cartoon typical of our measurements, exaggerated to illustrate the effect. The $x$-axis should indicate location in the figure (pixel coordinates), which is proportional to a measurement on the front ruler. The $y$-axis shows the measurement read from the middle ruler (true, physical height).

In the middle of Fig. 12, we show the image of a spherical bead, with prescribed constant velocity for illustrative purposes. In the physical coordinate system ( $y$-axis on top of Fig. 12), this bead falls 0.1 units between every frame. In the movie ( $x$-axis on top and $y$-axis on bottom of Fig. 12), the location gets mapped by the functional relationship shown. In areas of small slope, the bead stretches, with high slope it compresses.

For case A, the bead is centered at 0.75 in physical space, with radius 0.05 , through the mapping, but the vertical span of the bead in the image is compressed, as shown in frame 3 at bottom of Fig. 12. The true center of mass of the bead is mapped along the dashed line in the top of the figure and that mapped point is shown with the solid horizontal line in the central image. The $\times$ mark indicates the center of

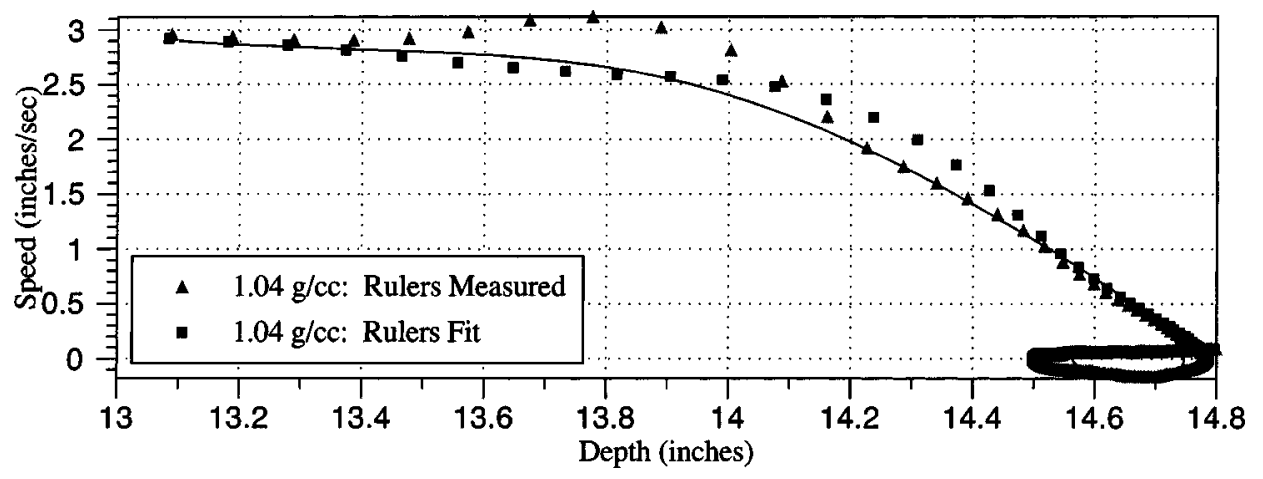

FIG. 11. Measured August bead velocity for $1.04 \mathrm{~g} / \mathrm{cm}^{3}$ bead; triangles showing measurement taken using measured, nonlinear pixel to physical coordinate mapping, squares using a linear fit to this nonlinear mapping; solid curve is output of nonlinear dynamical model with parameters $\beta_{0}$ $=0.06 / \mathrm{s}, \quad \beta_{\text {turb }}=0.94 / \mathrm{cm}, \quad z$ $=0.0445 \mathrm{~g}, \quad m=0.0751 \mathrm{~g}, \quad \omega$ $=0.15 \mathrm{~cm}$, see text. 

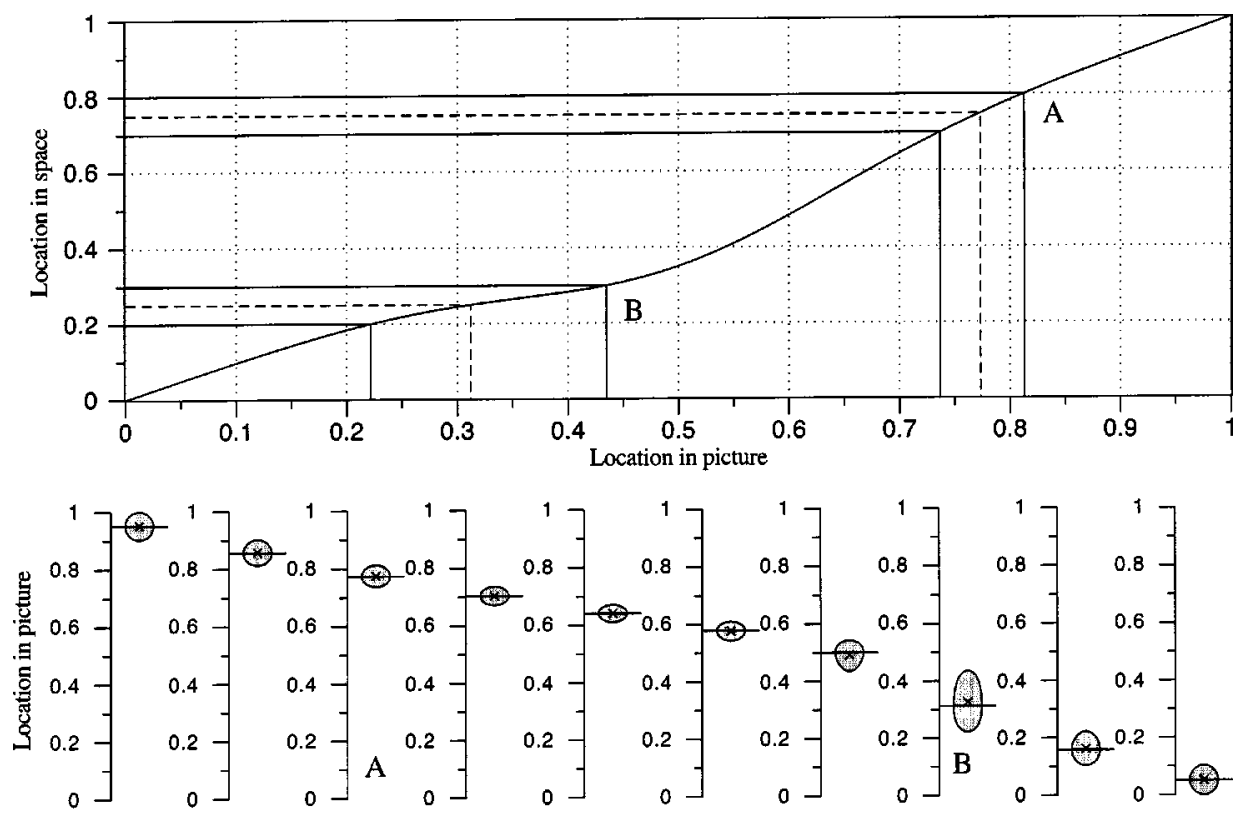

FIG. 12. Cartoon for constant moving bead through density transition. Top: showing physical coordinate to pixel coordinate mapping; middle: showing resulting bead image through mapping, and bottom showing ensuing velocity profile for (top) exact constant speed, (middle) velocity of the center of mass in pixel coordinates, and (bottom) the time derivative of the preimage of center of mass in pixel coordinates.

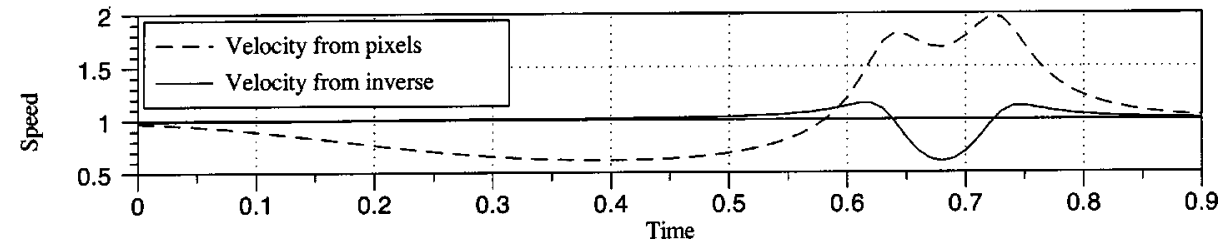

mass of the projection of the bead, computed from the picture.

In the bottom of Fig. 12 we show three computed velocity vs time plots; one for the true constant speed, one for the velocity computed from the center of mass in pixel coordinates, and lastly from the velocity computed by differentiating the preimage of that pixel coordinate (the technique used in the August study), see legend for ordering. From this picture its clear that the speed calculated from the pixels will underestimate the speed for the first six to seven time steps, and overestimate it for the last three. This explains the trend observed in the December 2002 data within the transition layer, along with the difference depicted in Fig. 11 for the August experiments. Obviously, a further improvement would be to first compute the preimage of the entire image, and perform all calculations and analysis upon that morphed coordinate system. Presumably this will yield a truer velocity profile which splits the difference between the data shown in Fig. 11. We leave this for future work. We emphasize that beyond the idealization of a constant, prescribed speed for this present discussion, and the selection of the mapping between physical and pixel coordinates (whose shape is extremely similar to the actual mapping), all subsequent conclusions are drawn from numerical calculations performed using the DataTank program, specifically the conclusion regarding the trends in velocity over and underestimation.

\section{REDUCED, COUPLED NONLINEAR MODEL: ENTRAINED MASS AND TURBULENT DIFFUSION}

Here we offer a simplified, reduced nonlinear dynamical system, generalizing the standard Newtownian force balance for a particle falling under gravity in a constant density fluid with known drag law to the case involving strongly stratified fluids. We exhibit the striking ability of this model to fit the complicated flight trajectories for the entire range of parameters using a single adjustable parameter, the entrained fluid mass.

To dynamically assess the motion of the sphere through this stratified fluid, it is necessary to model the entrained fluid as a dynamical degree of freedom, for without such a degree of freedom, the bead's motion will necessary yield a strictly monotonic connection between the top and bottom terminal velocities. This follows from physical principles, or from an elementary mathematical argument.

First, consider the relevant nonlinear dynamics associated with a fixed linear drag law moving through a monotonic, effective (reduced) gravitational field (we emphasize that the following mathematical argument easily generalizes to cases with nonlinear, monotonic drag laws which we will ultimately consider below, for ease in presenting the argument we first focus upon the linear case):

$$
\begin{aligned}
& m \frac{d^{2} X}{d t^{2}}+\alpha \frac{d X}{d t}=m g\left(\frac{\rho_{b}-\bar{\rho}(X(t))}{\rho_{b}}\right), \\
& \left.X\right|_{t=0}=0, \\
& \left.\frac{d X}{d t}\right|_{t=0}=v_{\text {term }}^{\text {top }}=\frac{m g\left(\rho_{b}-\bar{\rho}(0)\right)}{\alpha \rho_{b}} .
\end{aligned}
$$

Here, $X(t)$ denotes the bead's position as a function of time, initialized at the top of the tank. We will take $X$ to measure depth in the tank: zero will correspond to the top, and in- 
creasing $X$ corresponds toward the tank bottom. As such, the stably stratified density profile, $\bar{\rho}(X)$, will be a positive, increasing function (of increasing depth), $m, \rho_{b}$ denote the respective mass and density of the sphere, the coefficient, $\alpha$ is the (positive) drag coefficient for the sphere, and $g$ $=980 \mathrm{~cm} / \mathrm{s}^{2}$ is the positive gravitational acceleration constant. We will assume that the bead density, $\rho_{b}$ exceeds the tank density, $\bar{\rho}(X)$, for all depths. For the bead's velocity, $d X / d t$ to achieve a local minimum (which implies a nonmonotonic connection undershooting the minimum terminal velocity in the tank), we must have locally that the velocity's derivative should vanish, and further, the second derivative of the velocity should be positive at the minimum. We first argue that if this minimum velocity is to exist, it must be positive, for at the minimum the left and right-hand sides of Eq. (1) would have different signs. Moreover, with the minimum velocity being necessarily positive, we show it cannot exist with these dynamics: Computing the derivative of Eq. (1), and evaluating at the minimum, we have

$$
\left.\frac{d^{3} X}{d t^{3}}\right|_{X_{\min }}=-\left.g \frac{1}{\rho_{b}}\left(\frac{d \bar{\rho}(X)}{d X} \frac{d X}{d t}\right)\right|_{X_{\min }} .
$$

Since the derivative of the tank density field is strictly positive, and since the second derivative of the velocity must be positive at the minimum, we need the minimum velocity to be negative, but we just argued that this was not possible. Hence, these dynamics do not support a locally minimum velocity. To account for the missing degree of freedom associated with the entrained fluid, we consider the entrained mass of fluid to itself be a bead moving through the stratified fluid. We will viscously couple the sphere to the entrained fluid through the drag law by modifying the local sphere velocity to respect both the sphere's motion and the local motion of the ambient fluid. To do this, we will assume that the effect of the entrained, ambient fluid may be entirely replaced by a point mass with mass, $z$, located a fixed distance, $\omega$, above the bead, with velocity, $\eta(t)$, and density, $\rho(t)$. The falling sphere will be coupled to this idealized, dynamic fluid particle through the argument of the drag law in which the effective speed of the sphere will be measured relative to the speed of this fluid point mass. The dynamics of the fluid point mass will be self-consistently determined by its own force balance between the viscous drag force (relative to the falling sphere) and the buoyancy force. The idealized fluid particle will be able to develop a negative buoyancy (rising plume) self-consistently within this modelling approach. In Fig. 13, we give a schematic showing the relevant parameters in the model. For further detailed discussion of the parameter values used with this model, see below.

With these two extra degrees of freedom, we may write down the following coupled, four-dimensional, nonlinear, dynamical system:

$$
\begin{aligned}
& m \frac{d^{2} X}{d t^{2}}-F\left(2 \frac{d X}{d t}-\eta(t)\right)=m g\left(\frac{\rho_{b}-\bar{\rho}(X(t))}{\rho_{b}}\right), \\
& \frac{d \rho(t)}{d t}=\beta(\bar{\rho}(X(t))-\rho(t)),
\end{aligned}
$$

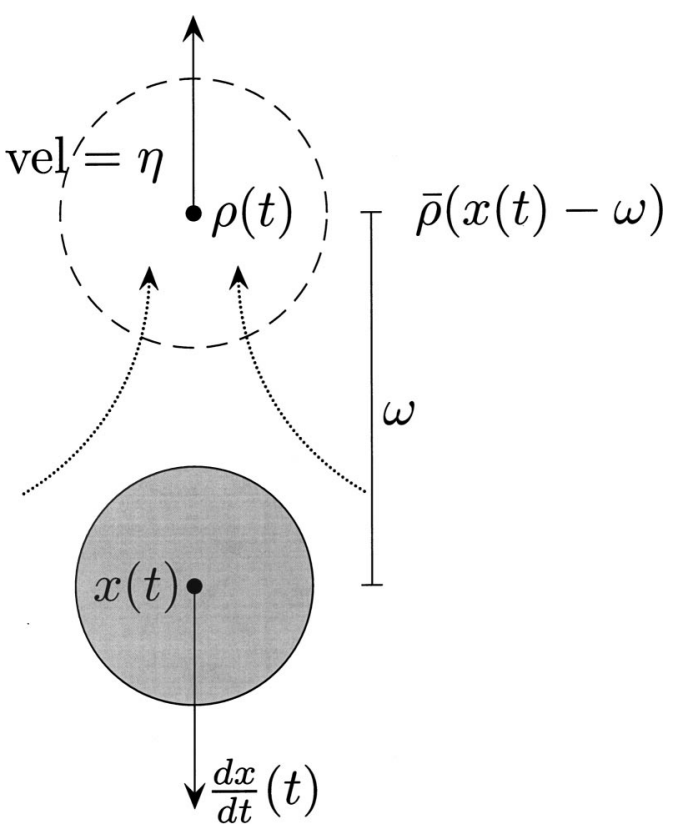

FIG. 13. Schematic depicting model parameters: Dynamic fluid replaced by idealized fluid particle, located a fixed distance, $\omega$, above the sphere, with dynamic velocity $\eta$, set by force balance between viscous drag and buoyancy force proportional to the density difference between the mixed fluid, $\rho(t)$, and the ambient fluid profile $\bar{\rho}(X(t)-\omega)$.

$$
z \frac{d \eta(t)}{d t}-F\left(\eta(t)-\frac{d X}{d t}\right)=z g\left(\frac{\bar{\rho}(X(t)-\omega)-\rho(t)}{\rho(t)}\right) .
$$

For initial conditions, the entrained fluid velocity will be set to the initial sphere velocity which is taken to be the terminal velocity of the top layer:

$$
\begin{aligned}
& X(0)=0, \\
& \eta(0)=\left.\frac{d X}{d t}\right|_{t=0}, \\
& \left.\frac{d X}{d t}\right|_{t=0}=-F^{-1}\left(\frac{m g\left(\bar{\rho}(0)-\rho_{b}\right)}{\rho_{b}}\right), \\
& \rho(0)=\bar{\rho}(0) .
\end{aligned}
$$

A short discussion regarding these dynamics is necessary here. First, we have assumed a general, odd, nonlinear, monotonic drag law $F$ on account of the nonlinear and monotonic reduction in slope exhibited in the drag data shown in Figs. 2 and 3, the inverse of this relation sets the terminal velocity of the top layer, which we take for the initial velocity. The new degrees freedom are hydrodynamically coupled to the falling sphere through the drag law. The drag experienced by a moving body is a function of the relative speed of the body as compared with its fluid surroundings. Inspection of Fig. 8 shows that the surrounding fluid actually becomes a degree of freedom in its ownright, and may move counter to the direction of motion of the solid body. Consequently, we augment our model to allow for this change in the relative speed between the fluid and entrained mass by modifying the argument of the nonlinear drag law above in Eq. (4), and symmetrically insist that the moving 
entrained mass be dragged by the falling body through the argument of the drag term in Eq. (6). There are a variety of ways that this explicit coupling may be inserted, we remark that the two appearing in the argument of the drag term in Eq. (4) simply insures that when the entrained mass and solid body are moving at the same speed, the solid body still experiences a hydrodynamic drag.

For most situations, outside of regions of strong density transition, the model has the entrained fluid moving at the same speed as the bead, and the argument reverts to that of the standard drag law, with argument set by the absolute speed relative to a fixed, inertial reference. Second, we let $z$ denote the mass of the entrained fluid, and this will be the only adjustable parameter adjusted to fit the data for different beads, all other parameters will be fixed at the outset. This mass is endowed with its own potential energy through buoyancy, which is modeled on the right-hand side of Eq. (6), where the location of the entrained fluid is idealized to be a point mass, located a fixed distance above the bead set by parameter $\omega$. We fix $\omega=0.15 \mathrm{~cm}$ for all subsequent simulations of the model. Regarding the mixing, in Eq. (5), we assume that the entrained fluid density mixes at a rate proportional to the local density difference between the entrained fluid and background fluid density, following a simple Newtonian cooling. We take proportionality constant, $\beta$ to in fact represent a turbulent diffusion, and depends upon the bead speed through

$$
\beta=\beta_{0}+\beta_{\text {turb }}\left|\frac{d X(t)}{d t}\right| .
$$

The coefficient $\beta_{0}$ should be taken as a very small constant of the order of the diffusivity of salt, $c\left(\kappa_{S} / L^{2}\right)$, where $\kappa_{S}$ $=0.000015 \mathrm{~cm}^{2} / \mathrm{s}$, and $c, L$ denote geometric factors. An exact theory for the shape, and volume of the entrained fluid does not exist, and consequently, the geometric factor will be selected empirically. The turbulent diffusivity, $\beta_{\text {turb }}$ also does not have a theoretical value, moreover, the precise functional dependence of the mixing coefficient upon the fluid flow may vary with the type of flow studied on account of the complexities associated with the closure problem of turbulence [see many references on passive scalar mixing for a complete mathematical account of just how complicated the situation is even for passive scalars both theoretically (Refs. 16-29), and experimentally (Refs. 30-34)]. For a successful modeling approach, it should be hoped that these mixing parameters do not change with experiment, and as such we fix the mixing parameters henceforth (except as otherwise mentioned) to be $\beta_{0}=0.06 / \mathrm{s}$, and $\beta_{\text {turb }}=0.94 / \mathrm{cm}$. We note that this particular value of $\beta$ is selected to match the 1.04 $\mathrm{g} / \mathrm{cm}^{3}$ bead, which in the bottom layer is most effected by this particular effect associated with the diffusivity of salt on account of the bead arrestment, subsequent reversal of motion, and ultimately slow decent (with minimal turbulent diffusion). Some additional remarks regarding the selection of these particular coefficients will be made in the following section in the context of the $1.04 \mathrm{~g} / \mathrm{cm}^{3}$ sphere.

Last, for the drag law, we utilize the power law fit between drag force and Reynolds number obtained in Fig. 3,
$F_{\text {drag }}(\mathrm{Re})=0.00064 \mathrm{Re}^{1.44} \mathrm{~g} \mathrm{~cm} / \mathrm{s}^{2}$ holding for our array of beads. To compute the drag, we calculate the Reynolds number locally in terms of the instantaneous velocity and assuming the bead is a perfect sphere.

We additionally point out that the model we have developed is idealized in many regards. Certainly, the fixed distance assumption between the sphere and the fluid point mass could be relaxed; however, to allow for this distance to vary would ultimately require developing an entrained fluid (reinitialization) replacement strategy because if the point mass moves too far away from the falling sphere, the hydrodynamic coupling will weaken as other ambient fluid fills in to replace the fluid. Further, there are presumably alternative hydrodynamic couplings to the viscous drag coupling employed here which may better represent the detailed mechanics of the forces at play as the sphere moves through the density transition layer. Nonetheless, as we shall see, the modeling approach we have taken does an excellent job in fitting the data, and does provide a very good first order description of the observed dynamics.

Modeling results: Tuned mixing, and quantified measures of entrainment: In this section we turn to fitting the experimentally measured trajectories depicted in Figs. 9 and 10. We first focus upon the case involving the $1.04 \mathrm{~g} / \mathrm{cm}^{3}$ bead, and then demonstrate the role of the turbulent diffusivity by focusing upon the $1.045 \mathrm{~g} / \mathrm{cm}^{3}$ bead. With the mixing coefficients then fixed, we turn to fitting the remaining trajectories through the adjustment of the single parameter, the entrained mass.

Using the nonlinear dynamical system given in Eqs. (4)(6), we adjust the mixing coefficients on the most complex trajectory, involving the $1.04 \mathrm{~g} / \mathrm{cm}^{3}$ sphere to match as best as possible the model with the data. Our approach will then be to fix the mixing parameters for all other trajectories, using the entrained mass, $z$, as the only adjustable parameter. Following this approach, we will extract a model estimate for the mass of the entrained fluid as a function of sphere density. Tuning the mixing coefficients on the $1.04 \mathrm{~g} / \mathrm{cm}^{3}$ December experiment yields, $\beta_{0}=0.06 / \mathrm{s}, \beta_{\text {turb }}=0.94 / \mathrm{cm}$. We emphasize that the selection of these parameters is important to correctly match the flight paths. In the bottom layer, the motion is extremely slow $(0.43 \mathrm{~cm} / \mathrm{s})$, so slow that the effect of the turbulent diffusion is negligible. We may quite accurately then tune the bare diffusivity, $\beta_{0}$, in this case to match the flight time: if the bare diffusivity is too small, the sphere does not travel far enough in the allotted time, too much bare diffusivity and it travels too far. The entrained mass coefficient is selected to best match the loop structure of the flight trajectory, which we find to be $z$ $=0.04792 \mathrm{~g}$ in this case. If the entrained mass is too large, the bead executes multiple loops, and never exists the transition layer, while if the entrained mass is too small, no loop structure, nor reversal of motion is evidenced in the trajectory. We stress that this particular case offers a rich trajectory demonstrating a wide range of regimes, and is extremely helpful in identifying the model parameters. Shown in Fig. 14 is the model output with these selected parameters superimposed upon the December measured $1.04 \mathrm{~g} / \mathrm{cm}^{3}$ bead tra- 


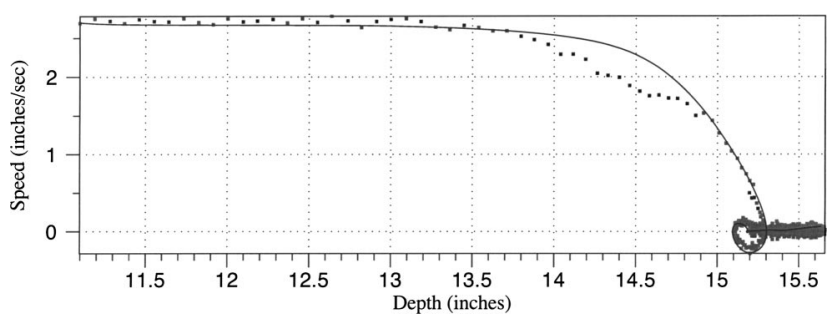

FIG. 14. Model output for the $1.04 \mathrm{~g} / \mathrm{cm}^{3}$ bead, matching December observation of speed vs depth, with $\beta_{0}=0.06 / \mathrm{s}, \beta_{\text {turb }}=0.94 / \mathrm{cm}, z=0.04792 \mathrm{~g}$, $m=0.0751 \mathrm{~g}, \omega=0.15 \mathrm{~cm}$.

jectory. The analogous case for the August measurement was shown in Fig. 11, with the only parameter difference being the entrained mass was selected to be $z=0.0445 \mathrm{~g}$.

One additional remark is in order regarding the December $1.04 \mathrm{~g} / \mathrm{cm}^{3}$ sphere. The reduction between the density difference between the bead and bottom layer fluid density (nearer to neutrally buoyant) leads to the additional long transient phenomena discussed above. We note that our model is not successful in accurately capturing this long transient phenomenon in the bottom layer for the $1.04 \mathrm{~g} / \mathrm{cm}^{3}$ sphere: for output times matching the experiment duration (35 s), the transient time scale of the nonlinear model to the terminal velocity of the bottom layer is shorter than the experimental observation for the parameters we have been able to study, as is evident by the tail of the model curve for this case (careful examination shows the model reverting to the limiting terminal velocity and departing from the measured trajectory toward the very end of the sequence). This is unique to the $1.04 \mathrm{~g} / \mathrm{cm}^{3}$ bead, the smallest density bead studied. To capture this subtlety will require a more careful modelling of the nature of the plume detachment, and entrained fluid replacement occurring as the bead penetrates the transition layer. An additional remark regarding the work of Srdic-Mitrovic et al., is necessary here. In Ref. 13 it is reported that all caudal fluid (entrained fluid from the upper layer) is disassociated from the falling sphere once the caudal plume has detached from the sphere. The extraordinarily long transient time scale which we observe for beads which are nearer to neutrally buoyant in the bottom layer is clear evidence that in such situations there is necessarily a small amount of caudal fluid still attached to the sphere. On account of the slow terminal velocity, this residual top layer fluid does not experience a Taylor dispersion capable of mixing this attached layer on faster than diffusive time scales [the time scale for mixing of this layer will be set by the small diffusivity of salt in water $\left.\left(0.000015 \mathrm{~cm}^{2} / \mathrm{s}\right)\right]$. We are in agreement with Srdic-Mitrovic et al., as regards the strong detachment of the majority of top layer entrained fluid, however, our experiments show that an additional small, but nonnegligible, amount of such caudal fluid does remain attached following the plume detachment.

We next document the importance of properly adjusting the turbulent mixing to match the data, we show the sample case of the $1.045 \mathrm{~g} / \mathrm{cm}^{3}$ bead next, with three different values of the turbulent mixing coefficient. In Fig. 15, we exhibit the output of the nonlinear model with entrainment mass ad-

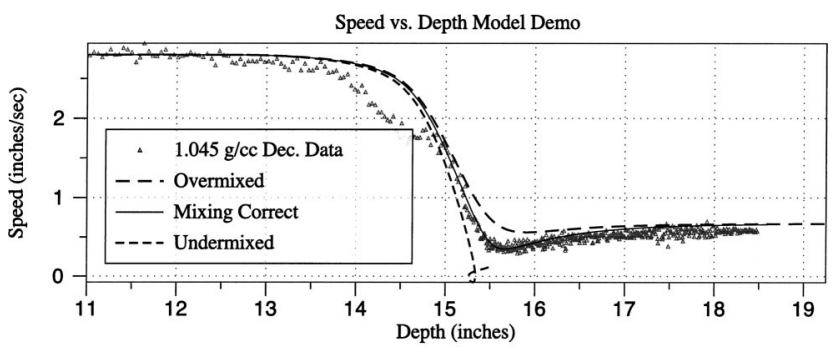

FIG. 15. Model output for $1.045 \mathrm{~g} / \mathrm{cm}^{3}$ bead, documenting the importance of mixing, with $z=0.029 \mathrm{~g}$, undermixed case with $\beta_{\text {turb }}=0.41 / \mathrm{cm}$, overmixed with $\beta_{\text {turb }}=1.5 / \mathrm{cm}$, and correct mixing with $\beta_{\text {turb }}=0.94 / \mathrm{cm}, m$ $=0.07 \mathrm{~g}, \omega=0.15 \mathrm{~cm}$, and $\beta_{0}=0.06 / \mathrm{s}$.

justed to $z=0.029 \mathrm{~g}$. The solid curve shows the correct value of the turbulent mixing coefficient, $\beta_{\text {turb }}=0.94 / \mathrm{cm}$, while the long dashed curve is under mixed, with $\beta_{\text {turb }}=0.41 / \mathrm{cm}$, and the short dashed curve has too much turbulent mixing, with $\beta_{\text {turb }}=1.5 / \mathrm{cm}$. Clearly, the capture of the nonmonotonic connection between the terminal velocities requires the careful tuning of the mixing coefficients: if the system mixes too strongly, the nonmonotonic connection is lost, while if the system is under mixed, the strength of the internal splash is too strong.

Last, we revert to the tuned mixing values $\beta_{0}=0.06 / \mathrm{s}$, $\beta_{\text {turb }}=0.94 / \mathrm{cm}, \omega=0.15 \mathrm{~cm}$, and adjust the entrained mass, $z$, to match the rest of the bead trials. The output of this study for the December data and August data are respectively shown in Figs. 16 and 17, superimposed atop of the actual measured velocity profiles. This documents the success of the nonlinear model in accurately modeling the flight trajectories for a range of bead densities through the adjustment of a single parameter, the entrained mass. The bead densities, masses, and range of acceptable values for the entrained mass, $z$, along with values for $z$ employed in Figs. 16 and 17 are given in Table I. For the acceptable values, we insist that the model depth be within $1 \mathrm{~cm}$ of the actual data at the end of the time integration, and further require that the depth of
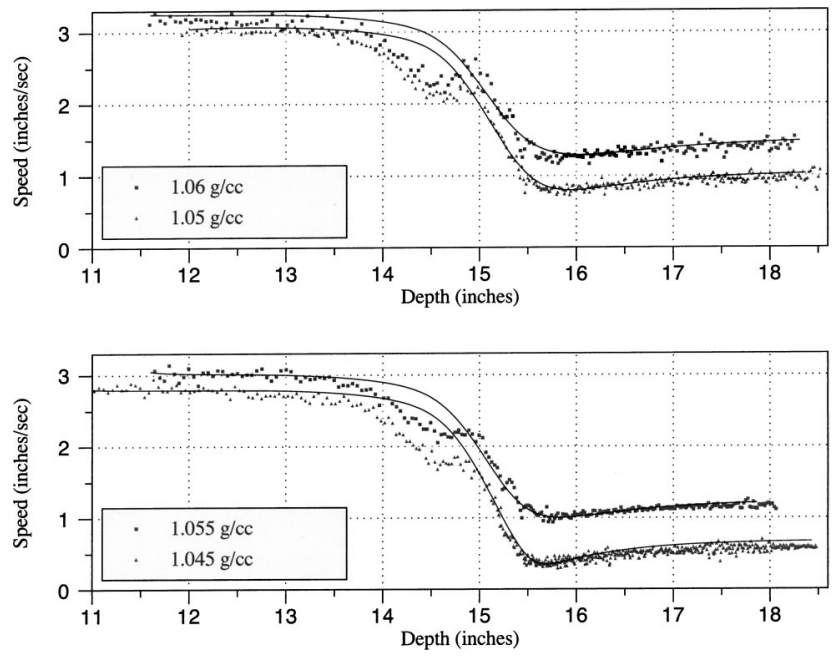

FIG. 16. Model output for December measurements $1.045 \mathrm{~g} / \mathrm{cm}^{3}$ through $1.06 \mathrm{~g} / \mathrm{cm}^{3}$ beads, $\omega=0.15 \mathrm{~cm}$, and $\beta_{0}=0.06 / \mathrm{s}$, and entrained mass, $z$, varies, see Table I. 

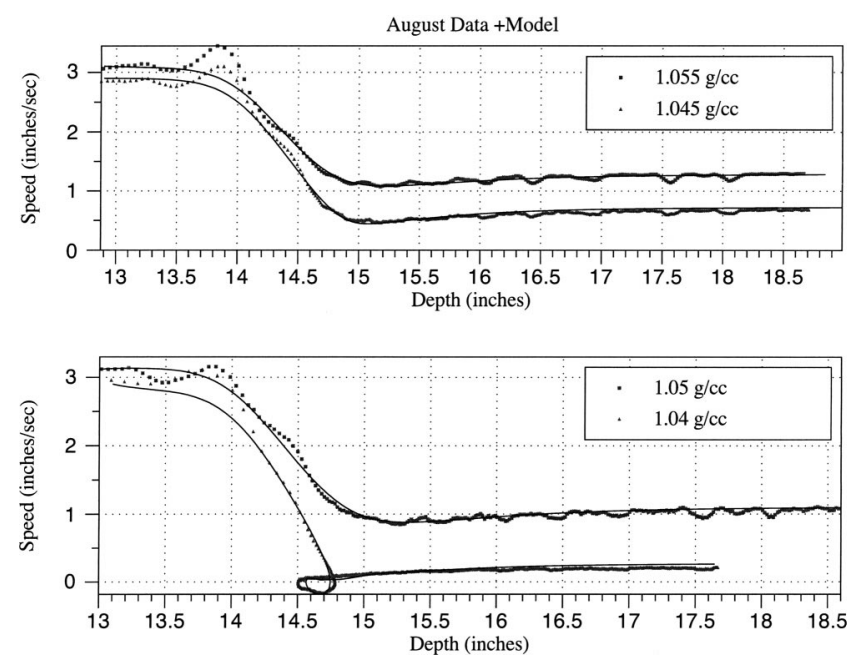

FIG. 17. Model output for August measurements $1.04 \mathrm{~g} / \mathrm{cm}^{3}$ through 1.055 $\mathrm{g} / \mathrm{cm}^{3}$ beads, $\omega=0.15 \mathrm{~cm}$ and $\beta_{0}=0.06 / \mathrm{s}$, and entrained mass, $z$, varies, see text.

the minimums between model and data be within $10 \%$ of each other. The beads do not have identical volume as is clear from Table I, and consequently there is not a clear ordering of the entrainment masses with respect to say bead density, and further study on this issue is merited. Last, returning to the issue of optical distortion within the transition layer, the improved method for measuring the velocity profile which we employed during the August study does show improved agreement between the model and measurement as compared with the more crude approach used during the December study. Further experiments and improved filtering will hopefully yield improved measuring techniques for such layered systems.

\section{CONCLUSIONS}

We have presented a carefully designed experiment illustrating the complex interactions occurring between a solid spherical body and stratified fluid which may ultimately yield transient levitation, and the reversal of motion of a falling body. Our experiments and modeling exhibit that the motion through this density transition is quite complex, with the interaction between the solid body and the entrained fluid becoming more dramatic as the sphere's density is adjusted to be closer to neutrally buoyant in the bottom layer. The

TABLE I. Bead densities, masses, ranges of acceptable entrained mass $(z)$ model parameter, and value of $z$ used in Figs. 16 and 17 .

\begin{tabular}{ccccc}
\hline \hline Date & Density $(\mathrm{g} / \mathrm{cc})$ & Mass $(\mathrm{g})$ & Entr. mass range $(\mathrm{g})$ & Entr. mass $(z)$ \\
\hline Dec & 1.040 & 0.0751 & $0.047-0.049$ & 0.0479 \\
Dec & 1.045 & 0.0705 & $0.026-0.029$ & 0.029 \\
Dec & 1.050 & 0.0761 & $0.034-0.038$ & 0.0377 \\
Dec & 1.055 & 0.0579 & $0.0214-0.0265$ & 0.024 \\
Dec & 1.060 & 0.0618 & $0.03-0.048$ & 0.04 \\
Aug & 1.040 & 0.0751 & $0.040-0.048$ & 0.0445 \\
Aug & 1.045 & 0.0705 & $0.026-0.029$ & 0.027 \\
Aug & 1.050 & 0.0761 & $0.031-0.038$ & 0.0317 \\
Aug & 1.055 & 0.0579 & $0.019-0.024$ & 0.0190 \\
\hline \hline
\end{tabular}

successful modeling of this behavior requires endowing the fluid with dynamic degrees of freedom to necessarily respect the potential energy associated with moving fluid to regions of differing densities. We have performed two careful studies to delineate and minimize the optical blur associated with the sharp change in optical refractive index in the vicinity of the density transition layer, and have shown that the careful measurement of the nonlinear mapping between the true depth measurement and imaged coordinates is key in obtaining accurate measurements within the transition layer. Last, we have documented the success of our reduced nonlinear dynamic system in modeling the observed velocity profiles through the adjustment of the model coefficient, the entrained mass.

Without doubt numerous extensions to the present study are planned, which include the study of the influence of the solid geometry (shape and scale) upon the observed motion. Certainly, the improved mathematical reduction of nonlinear models such as the one we studied here from the complete fluid equations is strongly desired.

Many scientific issues remain open to exploration. Most notably, can such an approach using nonlinear dynamical systems, and observations of falling solid bodies be used as an effective tool to better probe and understand environmentally important applications such as the mixing of pollution in both our atmosphere and oceans which possess numerous and frequent density transitions? But further, we hope that future exploration along the lines presented here may help to better understand at least some simplified aspects of how we interact with and modify our environment.

\section{ACKNOWLEDGMENTS}

R.M.M. is partially supported by a National Science Foundation Career Award DMS-97019242, and D.A. is partially supported through the Alfred Sloan Foundation. R.M.M. gratefully acknowledges helpful discussions from Roberto Camassa, Ray Goldstein, Ken McLaughlin, and thanks Joe Fernando for comments on the first draft, and suggesting Ref. 13. Additionally, R.M.M. thanks the Statistical and Applied Mathematical Sciences Institute (SAMSI) for partial support during the final stages of this work. All authors greatfully acknowledge the helpful suggestions of two anonymous reviewers which have improved the present study.

${ }^{1}$ P. Moin and J. Kim, “Tackling turbulence with supercomputers," Sci. Am. 276, 62 (1997).

${ }^{2}$ R. K. Adair, "The physics of baseball," Phys. Today 48 (5), 26 (1995).

${ }^{3}$ L. D. Landau and E. M. Lifshitz, Fluid Mechanics, 2nd ed. (Pergamon, New York, 1987).

${ }^{4}$ D. J. Tritton, Physical Fluid Dynamics, 2nd ed. (Oxford Science, Clarendon, 1995).

${ }^{5}$ H. J. S. Fernando, "Turbulent mixing in stratified fluids," Annu. Rev. Fluid Mech. 23, 455 (1991).

${ }^{6}$ J. J. Riley and M.-P. Lelong, "Fluid motions in the presence of strong stable stratification," Annu. Rev. Fluid Mech. 32, 613 (2000).

${ }^{7}$ D. L. Boyer and P. A. Davies, "Laboratory studies of orographic effects in rotating and stratified flows," Annu. Rev. Fluid Mech. 32, 165 (2000).

${ }^{8}$ B. R. Morton, G. I. Taylor, and J. S. Turner, "Turbulent gravitational convection from maintained and instantaneous sources," Proc. R. Soc. London, Ser. A 234, 1 (1956).

${ }^{9}$ H. B. Fischer, E. J. List, R. C. Y. Koh, J. Imberger, and N. H. Brooks, Mixing in Inland and Coastal Waters (Academic, New York, 1979). 
${ }^{10}$ J. S. Turner, Buoyancy Effects in Fluids (Cambridge University Press, New York, 1995).

${ }^{11}$ G. T. Csanady, "Turbulent diffusion in the environment," Geophysics and Astrophysics Monographs (Reidel, Dordrecht, 1973), Vol. 3D.

${ }^{12}$ L. H. Larsen, "Oscillations of a neutrally buoyant sphere in a stratified fluid," Deep-Sea Res. Oceanogr. Abstr. 16, 587 (1969).

${ }^{13}$ A. N. Srdic-Mitrovic, N. A. Mohamed, and H. J. S. Fernando, "Gravitational settling of particles through density interfaces," J. Fluid Mech. 381, 175 (1999).

${ }^{14}$ C. R. Torres, H. Hanazaki, J. Ochoa, J. Castillo, and M. Van Woert, "Flow past a sphere moving vertically in a stratified diffusive fluid," J. Fluid Mech. 417, 211 (2000).

${ }^{15}$ See EPAPS Document No. E-PHFLE6-16-708405 for an experimental video documentation of internal splash and motion reversal of a sphere falling through a sharply stratified fluid. A direct link to this document may be found in the online article's HTML reference section. The document may also be reached via the EPAPS homepage (http://www.aip.org.pubservs/epaps.html) or from ftp.aip.org in the directory /epaps/. See the EPAPS homepage for more information.

${ }^{16} \mathrm{G}$. I. Taylor, "Dispersion of soluble matter in solvent flowing slowly through a tube," Proc. R. Soc. London, Ser. A 219, 186 (1953).

${ }^{17}$ A. J. Majda and P. R. Kramer, "Simplified models for turbulent diffusion: Theory, numerical modelling, and physical phenomena," Phys. Rep. 314, 23 (1990).

${ }^{18}$ M. Chertkov, G. Falkovich, and I. Kolokolov, "Intermittent dissipation of a scalar in turbulence," Phys. Rev. Lett. 80, 2121 (1998).

${ }^{19}$ B. I. Shraiman and E. Siggia, "Lagrangian path integrals and fluctuations in random flow," Phys. Rev. E 49, 2912 (1994).

${ }^{20}$ J. C. Bronski and R. M. McLaughlin, "Rigorous estimates of the tails of the probability distribution function for the random linear shear model," J. Stat. Phys. 98, 897 (2000).

${ }^{21}$ E. Vanden-Eijnden, "Non-Gaussian invariant measures for the Majda model of decaying turbulent transport," Commun. Pure Appl. Math. 54, 1146 (2001).

${ }^{22}$ D. McLaughlin, G. Papanicolaou, and O. Pironneau, "Convection of mi- crostructure and related problems," SIAM (Soc. Ind. Appl. Math.) J. Appl. Math. 45, 780 (1985).

${ }^{23}$ A. J. Majda and R. M. McLaughlin, "The effect of mean flows on enhanced diffusivity in transport by incompressible periodic velocity fields," Stud. Appl. Math. 89, 245 (1993).

${ }^{24}$ A. Fannjiang and G. Papanicolaou, "Convection enhanced diffusion for periodic flows," SIAM (Soc. Ind. Appl. Math.) J. Appl. Math. 2, 333 (1994).

${ }^{25}$ A. J. Majda, "The random uniform shear layer: An explicit example of turbulent diffusion with broad tail probability distributions," Phys. Fluids A 5, 1963 (1993).

${ }^{26}$ R. T. Pierrehumbert, "Lattice models of advection-diffusion," Chaos $\mathbf{1 0}$, 61 (2000).

${ }^{27}$ Z. S. She and S. A. Orszag, "Physical model of intermittency in turbulence: Inertial range non-Gaussian statistics," Phys. Rev. Lett. 66, 1701 (1991).

${ }^{28}$ Ya. G. Sinai and V. Yakhot, "Limiting probability distributions of a passive scalar in a random velocity field," Phys. Rev. Lett. 63, 1962 (1989).

${ }^{29}$ J. Bonn and R. M. McLaughlin, "Sensitive enhanced diffusivities for flows with fluctuating mean winds: A two-parameter study," J. Fluid Mech. 445, 345 (2001).

${ }^{30}$ B. Castaing, G. Gunaratne, F. Heslot, L. Kadanoff, A. Libchaber, S. Thomae, X.-Z. Wu, S. Zaleski, and G. Zanetti, "Scaling of hard thermal turbulence in Rayleigh-Bénard convection," J. Fluid Mech. 204, 1 (1989).

${ }^{31}$ E. S. C. Ching, "Probabilities for temperature differences in RayleighBénard convection," Phys. Rev. A 44, 3622 (1991).

${ }^{32} \mathrm{~S}$. T. Thoroddsen and C. W. Van Atta, "Exponential tails and skewness of density-gradient probability density functions in stably stratified turbulence," J. Fluid Mech. 244, 547 (1992).

${ }^{33}$ P. Kailasnath, K. R. Sreenivasan, and G. Stolovitzky, "Probability density of velocity increments in turbulent flows," Phys. Rev. Lett. 68, 2766 (1992).

${ }^{34}$ L. C. Sparling, "Statistical perspectives on stratospheric transport," Rev. Geophys. 38, 417 (2000). 